\title{
IoT Telemonitoring System for COVID-19 Quarantine
}

\author{
Kobkiat Saraubon, Benchaphon Limthanmaphon
}

\author{
King Mongkut's University of Technology North Bangkok, Thailand
}

\begin{abstract}
The global COVID-19 outbreak has been spreading to 235 countries around the world. The quarantine can prevent the spread of disease. In this research, we developed and proposed an IoT telemonitoring system for quarantine employing IoT and mobile technologies. The system allows medical staff to monitor body temperature and track the location of every single quarantine person remotely by using a mobile device. When the body temperature is greater than the threshold $\left(38.0{ }^{\circ} \mathrm{C}\right)$, the system sends an alert to the medical staff via the mobile application. They can take the quarantined person to test for COVID-19. The average error of the system was less than $1 \%$. The evaluation results reveal that the users were satisfied with the system.
\end{abstract}

Keywords - COVID-19, IoT telemonitor, quarantine, non-contact temperature sensor.

\section{Introduction}

The current worldwide outbreak of COVID-19 (coronavirus) has been spreading to 235 countries around the world with 58,394,169 confirmed cases and 1,189,047 confirmed deaths reported to the World Health Organization (WHO).

DOI: $10.18421 /$ TEM101-13

https://doi.org/10.18421/TEM101-13

Corresponding author: Kobkiat Saraubon, King Mongkut's University of Technology North Bangkok, Thailand.

Email: kobkiat.s@sci.kmutnb.ac.th

Received: 22 October 2020.

Revised: 14 January 2021.

Accepted: 20 January 2021.

Published: 27 February 2021.

(cc) BY-NC-ND (C) 2021 Kobkiat Saraubon \& Benchaphon Limthanmaphon; published by UIKTEN. This work is licensed under the Creative Commons AttributionNonCommercial-NoDerivs 4.0 License.

The article is published with Open Access at www.temjournal.com
This virus causes a serious illness that affects respiratory in the human especially in older people and those with underlying medical problems like cardiovascular, disease, diabetes, chronic respiratory disease and cancer. Different kinds of symptoms have been diagnosed. The common signs and symptoms include fever, cough, body pain, and tiredness [1]. However, some infected people do not show any symptoms. An asymptomatic infected person is able to spread the coronavirus through his or her exhaled air. This virus can survive without a host on any surface for as long as nine days with the right temperature and humidity. The incubation period is between two to sixteen days. Therefore rapid diagnosis and rapid isolation is a crucial key to prevent transmission of this virus. Thus, social distancing, wearing a mask and quarantine are seriously taking into account to protect the family and community [2]. The quarantine can prevent the spread of disease that can occur before a person knows they are infected without any symptom. As stated by WHO, COVID-19 common symptoms are fever greater than $100.4^{\circ} \mathrm{F}$ ( or $38.0^{\circ} \mathrm{C}$ ), dry cough, fatigue, and shortness of breath [1]. Therefore, during the quarantine, the quarantined person needs to report his/her body temperature once or twice a day to the health care department or quarantine control center [3]. Thailand was an excellent example of fighting the coronavirus which has been ravaging the world since the beginning of this year, which was stated by WHO [4]. In Thailand, medical staffs are sent out to meet and measure the body temperature of the people in quarantine. Consequently, a shortage of medical staff could occur.

In this research, we developed and proposed a telemonitoring system based on IoT and mobile technologies. It can track the location and report the body temperature to the health care or quarantine control center. This paper is organized as follows. Section 2 reviews the relevant literature. Section 3 describes the design. Section 4 details the system architecture. Section 5 describes the usage scenarios. The results and evaluation are illustrated in section 6 . Finally, section 7 concludes the research. 


\section{Literature Review}

\subsection{Role of IoT in COVID-19}

The current cutting-edge of IoT and mobile technologies have an important role to play in COVID-19 struggling. They are able to minimize the need for medical staff interaction, risk, and prevent the spread of viruses. The IoT devices and services were used in many cases, including quarantine tracking, diagnosis, cleaning, and in-home infection reducing [5]. Nasajpour et al. [6], organized the role of IoT in COVID-19 and the future pandemics into three phases, including early diagnosis, quarantine, and after recovery. The first phase is important since the spreading of the virus can be controlled much better if the patient is diagnosed early. The IoT wearable devices such as wristbands, watches, and necklaces can be employed to capture information from the patients. During the quarantine phase, the patients need to be isolated in a hospital, provided place, or home. IoT devices are used to help the medical staff to monitor and track the patients as well as medical/delivery, robots, and drones are used to deliver food or medical supplies in order to reduce interaction between the patients and medical staff. In the last phase, which is after recovery, an effective solution such as social distancing has been implemented to ensure that the virus will not spread again. Wristbands, proximity devices, and RFID tags are the best approaches to ensure that people keep social distancing.

\subsection{Temperature Sensor}

Temperature is one of the most important physical parameters in many fields such as medical, industrial processes, manufacturing, engineering, agricultural and food industry. A temperature sensor is a device or module used to measure temperature from a particular source which indicates the temperature level in the form of analog or digital data read by other modules. Temperature sensors are classified into several types depending on sensing materials, operating principles, or physical types. According to the physical types, a temperature sensor consists of contact and non-contact temperature sensor [7].

A contact temperature sensor is required to be in physical contact with the surface of the investigated object since it uses thermal conduction to detect temperature. It can be used to detect solids, liquids, or gases. However, in contact temperature measurement dynamic errors are also observed. To acquire accurate measurement, the contact time between the object and the sensor has to be long enough to avoid inaccuracies offset by ambient temperature [8].
A non-contact temperature sensor measures a temperature changes by detecting the infrared energy emitted from an object. It can measure the temperature of all kind of materials and states including liquid, solid and gas [8]. The non-contact measurement using infra-red radiation provides low thermal response time and accurate temperature data without requiring direct contact or touch [9]. In recent years, non-contact measurement methods have been used for many areas such as manufacturing, electronics process, medical, health care, environmental monitoring, home automation, aerospace, and military applications [10]. There are many temperature sensors available for the IoT system. Table 1 shows low-cost temperature sensors for IoT [11], [12], [13].

Table 1. Temperature sensors [11] [12] [13]

\begin{tabular}{|c|c|c|c|c|}
\hline & \multirow{2}{*}{$\begin{array}{c}\text { Contact } \\
\text { DHT11 }\end{array}$} & $\begin{array}{c}\text { Digital } \\
\text { OneWire }\end{array}$ & $\begin{array}{c}0 \text { to } 50 \\
+/-2\end{array}$ & $6-12$ \\
\hline DHT22 & Contact & $\begin{array}{c}\text { Digital } \\
\text { OneWire }\end{array}$ & $\begin{array}{c}-40 \text { to } 80+/ \\
0.5\end{array}$ & $6-12$ \\
\hline LM35 & Contact & $\begin{array}{c}\text { Analog } \\
10 \mathrm{mV} /{ }^{\circ} \mathrm{C}\end{array}$ & $\begin{array}{c}-55 \text { to } 150 \\
+/-0.5\end{array}$ & $3-6$ \\
\hline TMP36 & Contact & $\begin{array}{c}\text { Analog } \\
10 \mathrm{mV} /{ }^{\circ} \mathrm{C}\end{array}$ & $\begin{array}{c}-40 \text { to } 125 \\
+/-1\end{array}$ & $7-12$ \\
\hline BMP180 & Contact & $\begin{array}{c}\text { Digital } \\
\text { IIC }\end{array}$ & $\begin{array}{c}0 \text { to } 65 \\
+/-0.5\end{array}$ & $5-10$ \\
\hline BMP280 & Contact & $\begin{array}{c}\text { Digital } \\
\text { IIC/SPI }\end{array}$ & $\begin{array}{c}-40 \text { to } 85 \\
+/-0.5\end{array}$ & $5-10$ \\
\hline DS18 B20 & Contact & $\begin{array}{c}\text { Digital } \\
\text { OneWire }\end{array}$ & $\begin{array}{c}-55 \text { to } 125 \\
+/-0.5\end{array}$ & $3-6$ \\
\hline MLX90614 & $\begin{array}{c}\text { Non- } \\
\text { contact IR }\end{array}$ & $\begin{array}{c}\text { Digital } \\
\text { IIC/PWM }\end{array}$ & $\begin{array}{c}-70 \text { to } 380^{\circ} \mathrm{C} \\
+/-0.5\end{array}$ & $<0.3$ \\
\hline
\end{tabular}

\subsection{Geolocation}

Geolocation refers to a technology used to identify the geographic location of an object, such as an Internet-connected mobile device, computer, IoT, or GPS-equipped device [14]. Geographic location data, also known as object location, consists of a set of geographic coordinates usually latitude and longitude (lat/long) to express and locate the object location on the Earth. Geolocating is an approach of determining the location/position of a device which can be classified into two methods - GPS and non-GPS methods [15].

The GPS method employs GPS technology to capture the latitude and longitude of a GPS-equipped device. It is the most precise method that generates the least errors, 3-5 meters for normal GPS depending on its built-in GPS sensor, and less than 10 centimeters for high-accuracy GPSs such 
as Global Navigation Satellite System (GNSS) and Real-Time Kinematic (RTK) [14]. Inside the GNSS, the principal operation is the same as normal GPS that the processing unit determines the position of the receiver by collecting information from a minimum of three satellites. But GNSS satellites transmit two carrier waves containing information to the Earth in the L-Band referred as L1 and L2 such as BDS, GLONASS, Galileo, and BeiDou. Real Time Kinematics (RTK) is another technology that provides high accuracy positioning system in the area of a base station which covers a service area around 10 to 20 kilometers.

For the non-GPS method, Fargas et al. [16], proposes a tracking system using LoRa technology without the use of GPS or GSM. Geolocation is calculated by applying a multilateration algorithm on the gateways timestamps from receiving packages. The entire system consists of an end-node, four gateways, and a server. The software includes Java application and MySQL database. The end-node sends data to the gateways located $5-10 \mathrm{~km}$ away using LoRaWAN protocol. Then, the gateways forward the packet's information including RSSI, working frequency, and others using a Message Queue Telemetry Transport (MQTT) to the application to determine the current location.

Google geolocation service [17] provides Geolocation API as a non-GPS geolocation method for Internet-connected devices. It returns the current location information of a device; latitude, longitude, and accurate radius. Google periodically collects Android-based mobile device locations using Android Location Services. The service gathers information from the GPS, Cell-ID, and Wi-Fi. Under the hood, Android-based mobile devices send publicly broadcast Wi-Fi access points Service set Identifier (SSID), Media Access Control (MAC) as well as current location to the Google server which will be used for the API. In other words, Google geolocation service collects location information reported from users' mobile devices as big data for reference. It then calculates and returns location data to the other devices when a device needs to know where it is. The more data points Google collects, the more accurate location system is. Most of the web browsers on computers and mobile devices support Geolocation API and the HTML5 Geolocation API but the IoT does not. However, the World Wide Web Consortium (W3C) has defined the W3C Geolocation API for Geolocation standard which is used by a server, client-side device, and IoT node. The standard defines scripts that execute in the client application. The location information generally determined by IP address, Wi-Fi access point, cellular site, radio-frequency identification (RFID), or device Global Positioning System (GPS). The accuracy of location depends on the types of sources. The GPS provide the best accuracy. Table 2 compares geolocation approaches.

Table 2. Geolocation approaches [16], [17]

\begin{tabular}{|l|c|c|}
\hline \multicolumn{1}{|c|}{ Description } & GPS & Non-GPS \\
\hline GPS module & Need & - \\
\hline Internet connection & - & Need \\
\hline Detection time & $10-120$ secs & $0.5-2$ secs \\
\hline Geolocation service & - & Need \\
\hline Accuracy & $3-5 \mathrm{~m}$ & $10-50 \mathrm{~m}$ \\
\hline
\end{tabular}

\subsection{Microcontroller for IoT}

IoT technology has made significant progress towards developing smart devices. This enables components to sense, communicate, interact, and collaborate with other physical things over the Internet. The heart of IoT systems is the main processor unit (microcontroller: MCU) that processes data and executes software. There are numbers of MCU available for IoT applications [18]. Saraubon et al. [19], developed an asset tracking system based on IoT and mobile technologies. The system architecture was comprised of NFC identification tags, an IoT module, a web server, and a mobile application. The IoT module employed ESP32 MCU. The proposed system allows employees to track and report asset status. Dada et al. [20], proposed a remote weather monitoring system. The ATmega328P mounted on Arduino Uno development board was used in the research. The whole system comprised LDR, temperature humidity sensor and, real-time clock module (RTC).

\section{System Analysis and Design}

\subsection{Stakeholders}

The system is designed for telemonitoring system for quarantine which is dealing with stakeholders according to their concerns as follows:

- Quarantine people: people who are kept in quarantine for fourteen days. During the quarantine period, they need to report the status to the medical center at least once a day. The status data includes user ID, body temperature, and their current location.

- Medical staff: who control and keep checking the status of the quarantine people day-by-day. If anyone in the report has a body temperature of $38.0^{\circ} \mathrm{C}$ or higher, they will take the quarantine people to test for COVID-19. 


\subsection{Requirement Gathering and Analysis}

The requirements were analyzed and determined from the information gathered from the staff and the experts involved the virus situation. This was obtained by the in-depth interviews under the requirement engineering and the IoT framework [21] [22]. The following are the conclusions with regard to the specified functional requirements in the terms of actions and functions:

- The IoT module has to detect and report the body temperature and location of the quarantine people. The temperature sensor should be a noncontact type and it should take less than two seconds to capture the body temperature. The sensor should produce an error less than $1 \%$ at room temperature.

- The medical staffs are able to monitor the body temperature and the location of the quarantine people. If the body temperature is greater than the threshold (usually $38.0^{\circ} \mathrm{C}$ ), the system should alert, thus, the medical staff will take that person to test for COVID-19 infection. The mobile application can navigate to where the suspect person lives.

\section{System Architecture}

This section describes the system architecture and the components that employ the IoT and mobile technologies. Figure 1 depicts the proposed system architecture. It is comprised of an IoT-based device a server, and clients.

\subsection{IoT module}

An IoT telemonitoring module illustrated in Figure 1 and 3 is used to detect the body temperature of a person and determine its current location. The module comprises four main components: MCU, non-contact temperature sensor, OLED display, and distance sensor.
Non-contact temperature sensor: a Melexis MLX90614 was used as a temperature sensor. It is an infrared thermo-sensor designed for non-contact temperature sensing. Internally, the MLX90614 is comprised of two main components, an infrared thermopile detector and a signalconditioning application processor with an internal 17-bit analog-to-digital converter (ADC) and a powerful digital signal processor (DSP) [12].

The MLX90614 comes in an industry-standard TO-39 package integrated with pull-up resistors as shown in Figure 2. It measures and produces two

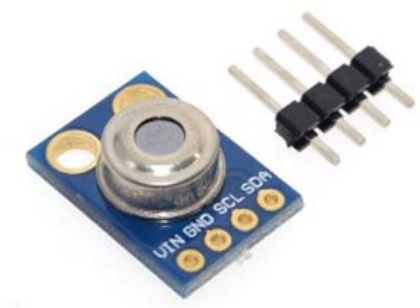

Figure 2. Non-contact temperature sensor

temperatures, an object and ambient temperature. The object temperature is the non-contact measurement being sensed, while the ambient temperature measures the temperature on the body of the sensor. The module interface supports PulseWidth modulation (PWM) and Inter-Integrated Circuit (I2C). The sensor provides accurate outputs of $0.14^{\circ} \mathrm{C}$ and $0.02^{\circ} \mathrm{C}$ for PWM and I2C interface respectively. The sensor is factory calibrated in the temperature ranges between -40 to $85^{\circ} \mathrm{C}$ for the ambient temperature and -70 to $382.2^{\circ} \mathrm{C}$ for the object temperature (non-contact surface measurement). In general, the sensor provides a standard accuracy of $0.5^{\circ} \mathrm{C}$ since the measured value is from the average temperature of all objects in the window view of the sensor.

MCU: WeMos ESP32 OLED was used as the MCU and display. It is a development board equipped with a 128x64 SSD1306 OLED display.

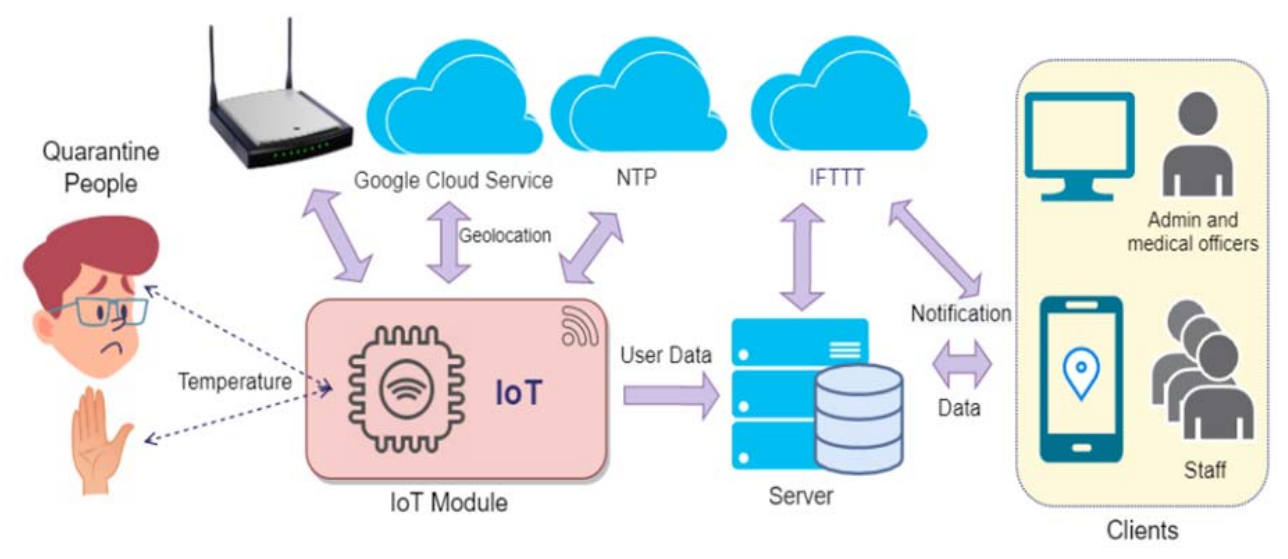

Figure 1. System Architecture 
The board is designed for a mobile system, wearable electronics, and the Internet of Things (IoT) applications. The OLED display communicates with the ESP32 chip via I2C communication protocol (ESP32 GPIO5=SDA; GPIO4=SCL). The main control chip employs a $240 \mathrm{MHz}$ dual-core processor. It comes with $\mathrm{Wi}-\mathrm{Fi}$ and Bluetooth (Bluetooth dual mode integrated traditional and BLE low-power Bluetooth). Table 3 shows the pin connection between the modules.

Table 3. Pin connection

\begin{tabular}{|c|c|c|}
\hline ESP32 PIN & I2C module & Description \\
\hline GND & Ground & $0 \mathrm{~V}$ \\
\hline 3.3V & Power & $3.3 \mathrm{~V}$ \\
\hline GPIO5 & SDA & Serial Data \\
\hline GPIO4 & SCL & Serial Clock \\
\hline
\end{tabular}

Distance sensor: the sensing temperature may inaccurate due to the distance between the object and the non-contact temperature sensor. In this research, a Time-of-Flight VL53L0X distance sensor was used to provide the system working as an auto-trigger mode. When the users aim and approach the temperatures sensor to their forehead or palm at a certain distance, the module reads the temperature and reports to the server automatically. fast mode at $400 \mathrm{kHz}$ standard which quick enough to trigger the IoT module.

There are three I2C interface modules connected to the main MCU (ESP32) on the same bus; an MLX90614 non-contact temperature sensor, VL53L0X Time of Flight distance sensor, and SSD1306 OLED display. Each module is a slave device that has its own unique address, 0x3C, 0x29, and 0x5A for MLX90614 sensor module, VL53L0X distance sensor, and SSD1306 OLED display respectively.

The I2C communication pins for ESP32 are pin number 5 (Serial Data: SDA) and pin number 4 (Serial Clock: SCL). To enable the OLED display, it has to initialize using display $(0 \times 3 c, 5,4)$. The schematic diagram is shown in Figure 3. The IoT module prototype is shown in Figure 4.

\subsection{Server and Services}

The application and database server receives information from the IoT modules and handle the queries requested by the client device. The information includes user ID, the body temperature, and the location of the user. The system employs three services, including Google geolocation, IFTTT, and NTP.

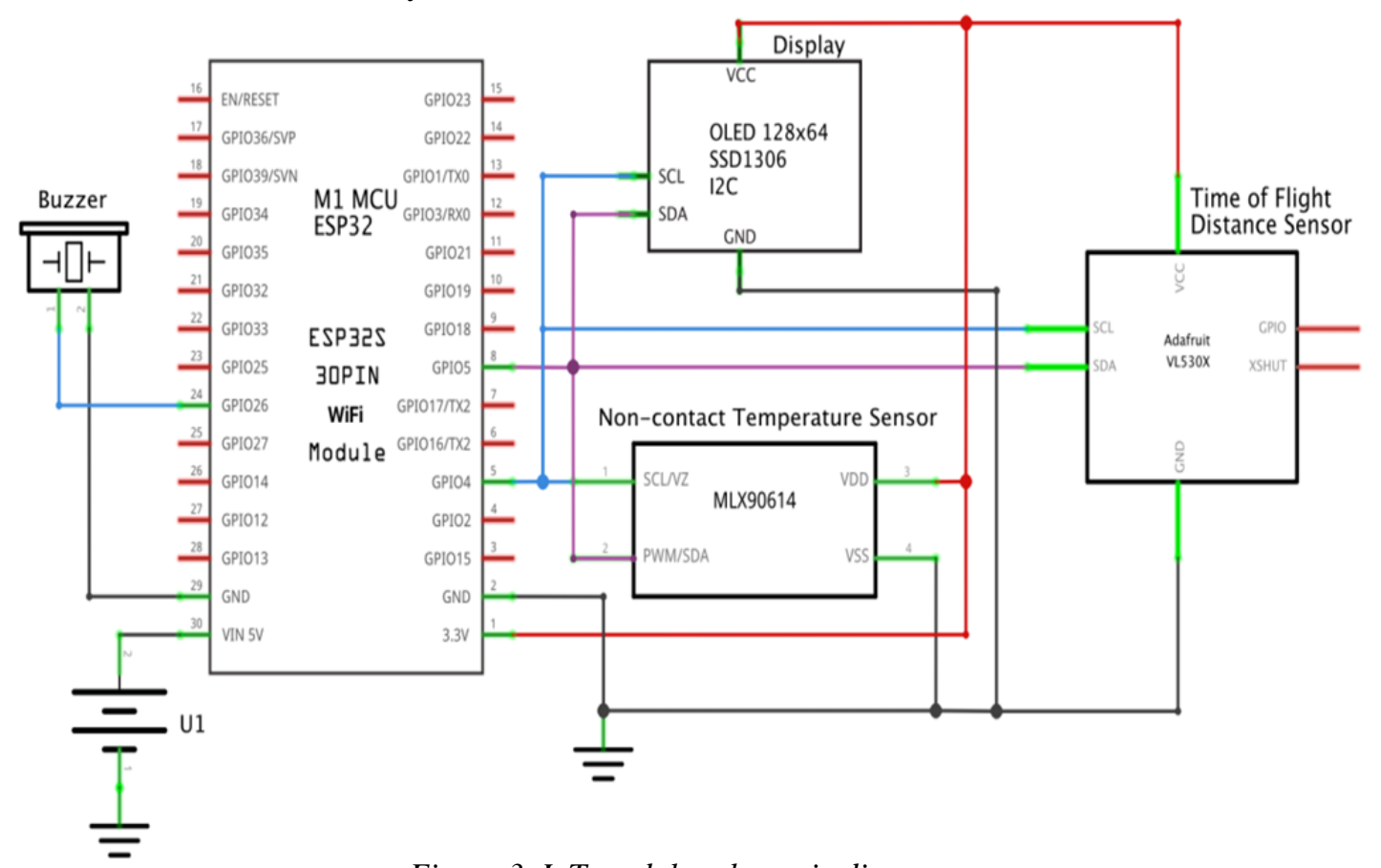

Figure 3. IoT module schematic diagram

The Time-of-Flight VL53L0X distance sensor employs FlightSense laser technology to measure the absolute distance from the sensor to the surface of the target object quickly and accurately. The interface on the VL53L0X is compatible with the I2C
The Google geolocation service is used to determine the current location of the quarantine person. The first procedure is to register and get a token for the service. The IFTTT is used for sending alerts to the medical staff while NTP is used for time reference. 


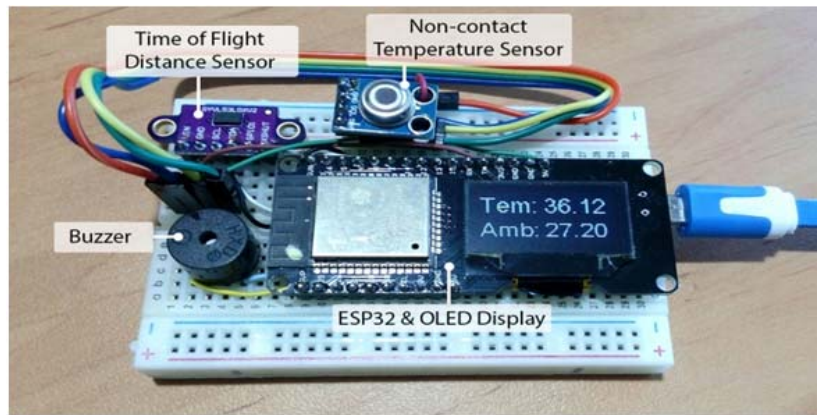

Figure 4. IoT module prototype

\subsection{Clients}

Computers and mobile devices are the client-side allowing the medical staff to monitor body temperature and location of the people in quarantine remotely. The mobile application running on mobile devices was developed using Android Studio; the official Integrated Development Environment (IDE) for Android devices. The device receives an alert when the temperature is greater than the threshold.

\section{Scenario}

Figure 5 and the following descriptions describe the usage scenario for suspect people and the medical staff.

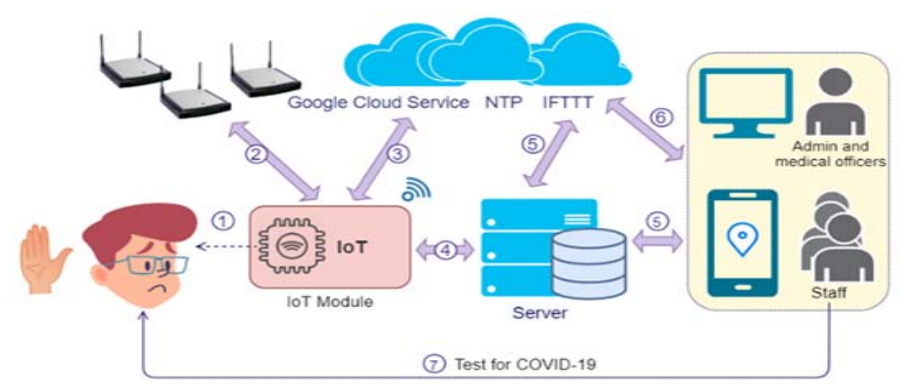

Figure 5. Usage scenario

i. A suspect or quarantine person measures his/her body temperature using the IoT telemonitor module by approaching the module to his/her forehead or palm hand;

ii. The Iol telemonitor module reads temperature and scans Wi-Fi network nearby to get SSIDs information;

iii. The IoT module sends SSIDs to the Google Cloud Service then gets the location data of the IoT module (latitude, longitude, and accuracy of the radius base);

iv. User ID, temperature, and geolocation location are sent to the application server;

v. Application server sends notification data to IFTTT service. Client devices allow admin and staff to monitor each suspect people data;

vi. When the temperature is greater than the threshold $\left(38.0^{\circ} \mathrm{C}\right)$, the system sends an alert to the staff. They will take the quarantine person to test for COVID-19.

\section{Results}

This section describes the results of the test and evaluation. The system was first tested by the developers. Then, it was tested and evaluated by medical staff and volunteers.

\subsection{Temperature}

We tested the system on three circumstances: onehundred objects, ambient temperatures, and human body temperatures. All cases were compared to a calibrated thermometer. The thermal response of the module was less than 0.5 second, which means that the module needed less than 0.5 second to read the body temperature. Table 4 presents the result of the comparisons. The average error was less than $1 \%$.

Table 4. Comparison table $(N=100)$

\begin{tabular}{|c|c|c|c|}
\hline Temperature & $\begin{array}{c}\text { Average } \\
\text { Error (\%) }\end{array}$ & SD & $\begin{array}{c}\text { Max } \\
\text { Error (\%) }\end{array}$ \\
\hline Ambient & 0.91 & .0014 & 0.97 \\
\hline Object & 0.92 & .0009 & 1.02 \\
\hline Human-body & 0.95 & .0007 & 1.01 \\
\hline
\end{tabular}

\subsection{Location}

We tested the system with fifty volunteers located on different locations. The geolocations determined by the system were compared with the actual locations. The average error and SD were 12.1 meters and 1.73 respectively. The results showed that the geolocation determined by the IoT module is accurate enough for the medical staff to locate and track the quarantine people.

On the mobile application, there was a list of suspected people shown in Figure 6. It showed the information of each one; body temperature, date time of last measured. The quarantine person having the highest temperature is on the top of the list. The medical staffs are able to tap on each item to see temperature history (time-series data) or location and use a navigation system to navigate to the quarantine person location as seen in Figure 6. If the temperature reaches the threshold, a notification is sent to the medical staff to the email and instance messengers. 

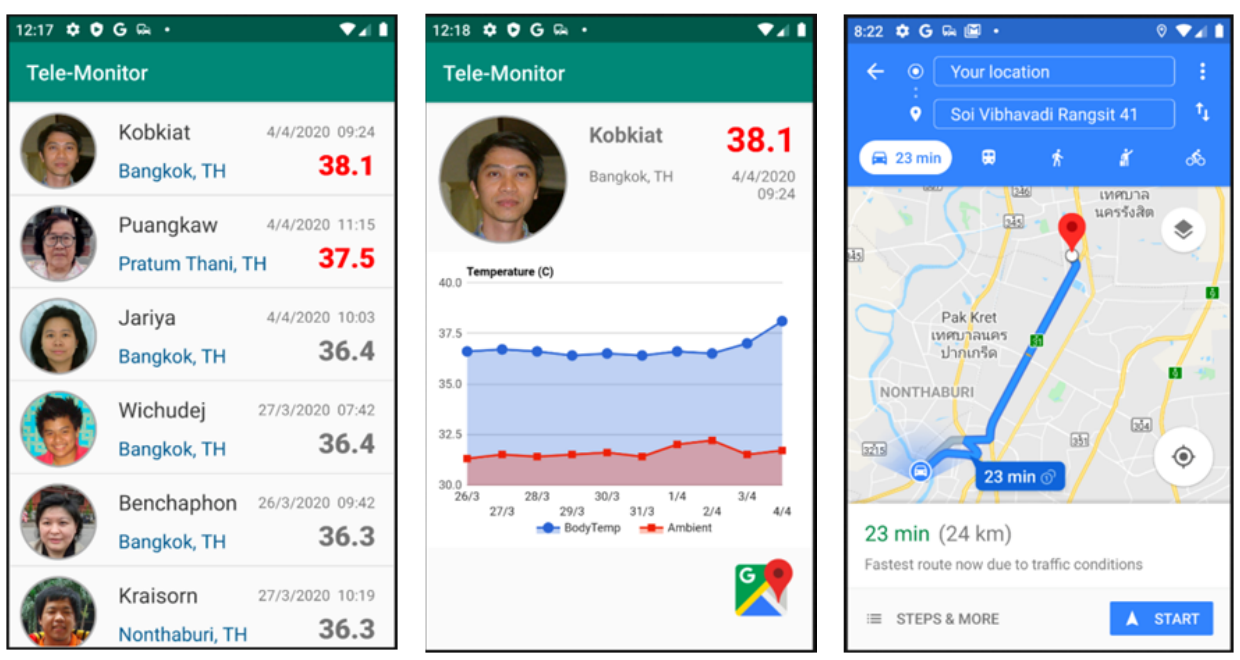

Figure 6.UI on the mobile application

\subsection{Evaluation}

The system was evaluated by two groups. They were fifteen medical staffs and thirty volunteers. Both of them had experience in system development. The evaluation metrics focused on four dimensions including functionality, usability, security and privacy. The functionality covers accuracy, performance, and interoperability. The usability focuses on learnability, operability and usefulness while the security and privacy also includes stability. The data were analyzed to determine the mean and standard deviation. The results evaluated by the medical staff shown in Table 5 reveals that the functionality $(M=4.54, S D=0.51)$, usability $(M=4.50$, $S D=0.52)$, and security and privacy $(M=4.57$, $S D=0.52$ ), were evaluated as the highest level while the results given by the users indicates that the functionality $(M=4.53, S D=0.51)$, usability $(M=4.58$, $S D=0.51)$, security and privacy $(M=4.47, S D=0.51)$, and overall were evaluated as the highest and a high level.

Table 5. The results of the evaluation

\begin{tabular}{|c|c|c|c|}
\hline Dimension & Mean & SD & Meaning \\
\hline \multicolumn{4}{|c|}{ Medical staff } \\
\hline 1. Functionality & 4.54 & 0.51 & Highest \\
\hline 2. Usability & 4.50 & 0.52 & Highest \\
\hline $\begin{array}{l}\text { 3. Security and } \\
\text { privacy }\end{array}$ & 4.57 & 0.51 & Highest \\
\hline 4. Overall & 4.65 & 0.53 & Highest \\
\hline \multicolumn{4}{|c|}{ Users } \\
\hline 1. Functionality & 4.53 & 0.51 & Highest \\
\hline 2. Usability & 4.58 & 0.52 & Highest \\
\hline $\begin{array}{l}\text { 3. Security and } \\
\text { privacy }\end{array}$ & 4.47 & 0.51 & High \\
\hline 4. Overall & 4.55 & 0.55 & Highest \\
\hline
\end{tabular}

\section{Conclusion}

During the outbreak crisis, COVID-19, for example, quarantine can prevent the spread of disease that can occur before a person knows they are infected without any symptom. A quarantine person should stay at home or provided place for fourteen days, separate from others, self-monitor, and report his/her body temperature once or twice a day to the health care department. In Thailand, medical staffs are sent out to meet and measure the body temperature of the people in quarantine. Consequently, a shortage of medical staff can occur. In this study, we developed and proposed a telemonitoring system based on IoT and mobile technologies. It can track the location and report the body temperature to the health care or quarantine control center. The medical staffs are able to monitor and track every single quarantine person by using a mobile device. When the temperature is greater than the threshold $\left(38^{\circ} \mathrm{C}\right)$, the system sends an alert to the medical staff via the mobile application. They will take the quarantine suspect person to test for COVID-19. The system could minimize the need for medical staff interaction and the risk of virus infection. The thermal response was less than 0.5 second which means that the module needed less than 0.5 second to read the body temperature while the average error was less than $1 \%$. The evaluation results reveal that the users were satisfied with the system. 


\section{References}

[1]. Wu, Y. C., Chen, C. S., \& Chan, Y. J. (2020). The outbreak of COVID-19: An overview. Journal of the Chinese Medical Association, 83(3), 217.

[2]. Chiu, N. C., Chi, H., Tai, Y. L., Peng, C. C., Tseng, C. Y., Chen, C. C., ... \& Lin, C. Y. (2020). Impact of Wearing Masks, Hand Hygiene, and Social Distancing on Influenza, Enterovirus, and All-Cause Pneumonia During the Coronavirus Pandemic: Retrospective National Epidemiological Surveillance Study. Journal of Medical Internet Research, 22(8).

[3]. Chung, Y. T., Yeh, C. Y., Shu, Y. C., Chuang, K. T., Chen, C. C., Kao, H. Y., ... \& Ko, N. Y. (2020). Continuous temperature monitoring by a wearable device for early detection of febrile events in the SARS-CoV-2 outbreak in Taiwan, 2020. Journal of Microbiology, Immunology, and Infection, 53(3), 503.

[4]. WHO. (2020). WHO Director-General's closing remarks at the World Health Assembly.

Retrieved from: https://www.who.int/directorgeneral/speeches/detail/who-director-general-sclosing-remarks-at-the-world-health-assembly---13november-2020 [accesse: 20 November 2020].

[5]. Kumar, K., Kumar, N., \& Shah, R. (2020). Role of IoT to avoid spreading of COVID-19. International Journal of Intelligent Networks, 1, 32-35.

[6]. Nasajpour, M., Pouriyeh, S., Parizi, R. M., Dorodchi, M., Valero, M., \& Arabnia, H. R. (2020). Internet of Things for Current COVID-19 and Future Pandemics: an Exploratory Study. Journal of Healthcare Informatics Research, 4, 325-364.

[7]. Jin, G., Zhang, X., Fan, W., Liu, Y., \& He, P. (2015). Design of Non-Contact Infra-Red Thermometer Based on the Sensor of MLX90614. Open Automation and Control Systems Journal, 7, 8-20.

[8]. Michalski, L., Eckersdorf, K., Kucharski, J., \& McGhee, J. (2001). Dynamic Temperature Measurement. Proceedings Tempus Summer School "Sensor Construction, 326-351.

[9]. Zhang, J. (2017). Development of a Non-contact Infrared Thermometer. Advances in Engineering Research, 153, 308-312.

[10]. Ning, B., \& Wu, Y. (2010, December). Research on Non-contact infrared temperature measurement. In 2010 International Conference on Computational Intelligence and Software Engineering (pp. 1-4). IEEE.
[11]. Schwartz, M. (2016). Internet of Things with ESP8266. Packt Publishing Ltd.

[12]. Marques, G., \& Pitarma, R. (2019). Non-contact Infrared Temperature Acquisition System based on Internet of Things for Laboratory Activities Monitoring. Procedia Computer Science, 155, 487494.

[13]. Bhadani, P., \& Vashisht, V. (2019, January). Soil moisture, temperature and humidity measurement using Arduino. In 2019 9th International Conference on Cloud Computing, Data Science \& Engineering (Confluence) (pp. 567-571). IEEE.

[14]. Li, X., Lv, H., Ma, F., Li, X., Liu, J., \& Jiang, Z. (2019). GNSS RTK positioning augmented with large LEO constellation. Remote Sensing, 11(3), 228.

[15]. Cornwall, J., Case, K., Callan, C., Despain, A., \& Dyson, F. (2002). Non-GPS methods of geolocation. Mitre Corp Mclean Va Jason Program Office.

[16]. Fargas, B. C., \& Petersen, M. N. (2017, June). GPSfree geolocation using LoRa in low-power WANs. In 2017 global internet of things summit (Giots) (pp. 1-6). IEEE.

[17]. Kavitha, D., \& Ravikumar, S. (2020). Designing an IoT based autonomous vehicle meant for detecting speed bumps and lanes on roads. Journal of Ambient Intelligence and Humanized Computing, 1-10.

[18]. Char, K. (2015). Internet of things system design with integrated wireless MCUs. Silicon Labs, ARM, Tech. Rep

[19]. Saraubon, K., Chinakul, P., \& Chanpen, R. (2019, December). Asset Management System using NFC and IoT Technologies. In Proceedings of the 2019 3rd International Conference on Software and $e$ Business (pp. 124-128).

[20]. Dada, E. G., Joseph, S. B., Mustapha, D., \& Hena, B. I. (2018). Microcontroller Based Remote Weather Monitoring System. vol, 5, 276-287.

[21]. Pressman, R. S., \& Maxim, B. (2015). Software Engineering: A Practitioner's Approach 8th Edition (8 ed.). New York, USA: McGraw-Hill.

[22]. Uviase, O., \& Kotonya, G. (2018). IoT architectural framework: connection and integration framework for IoT systems. arXiv preprint arXiv:1803.04780. 